\title{
ON THE INTERSECTION OF HERMITIAN SURFACES
}

\author{
L. GIUZZI
}

\begin{abstract}
We provide a description of the configuration arising from intersection of two Hermitian surfaces in $\mathrm{PG}(3, \mathrm{q})$, provided that the linear system they generate contains at least a degenerate variety.
\end{abstract}

\section{INTRODUCTION}

In [2], the seven point-line configurations arising from the intersection of two Hermitian curves are described and a classification of Hermitian pencils yielding a given configuration is provided. In [3] it has been shown that these configurations are projectively unique and their full collineation group has been determined. Given two Hermitian varieties $\mathcal{H}_{1}$ and $\mathcal{H}_{2}$, their intersection $\mathcal{E}$ is just the base locus of the $\mathrm{GF}(\sqrt{\mathrm{q}})$-linear system they generate, namely

$$
\Gamma\left(\mathcal{H}_{1}, \mathcal{H}_{2}\right)=\left\{\mathcal{H}_{1}+\lambda \mathcal{H}_{2}: \lambda \in \mathrm{GF}(\sqrt{\mathrm{q}})\right\} .
$$

In this paper we determine the size of such an intersection depending on the number of degenerate varieties in $\Gamma$ and describe the actual point-line configurations arising in the 3 -dimensional case, provided that $\Gamma$ contains at least a degenerate surface.

\section{INTERSECTION NUMBERS}

The set of all singular points of a Hermitian variety $\mathcal{H}$ is a subspace $\operatorname{rad} \mathcal{H}$, the radical of $\mathcal{H}$. We recall that the rank of a Hermitian variety in $\mathrm{PG}(\mathrm{n}, \mathrm{q})$ is the number $r=n+1-\operatorname{dim} \operatorname{rad} \mathcal{H}$.

Let now $\mathcal{H}_{1}$ and $\mathcal{H}_{2}$ be two Hermitian hypersurfaces of PG(n, q) and denote by $r_{i}$ the number of varieties of rank $i$ in the $\mathrm{GF}(\sqrt{\mathrm{q}})$-pencil $\Gamma$ they generate. We shall call the list $\left(r_{1}, \ldots, r_{n}\right)$ the rank sequence of $\Gamma$.

It has been observed in [2] that the cardinality of the base locus $\mathcal{E}$ of $\Gamma$ depends only on its rank sequence. In fact the considerations provided in [2] about the 2-dimensional case may be generalised to arbitrary dimension $n$, as it has been done in [4].

Proposition 1. The rank sequence $\left(r_{1}, \ldots, r_{n}\right)$ of a pencil $\Gamma$ of Hermitian varieties in $\mathrm{PG}(\mathrm{n}, \mathrm{q})$ satisfies the inequality

$$
\sum_{i=1}^{n}(n-i+1) r_{i} \leq n+1 .
$$

Since, see [1], the total number of points of the non-degenerate Hermitian hypersurface $\mathcal{H}$ of $\mathrm{PG}(\mathrm{n}, \mathrm{q})$ is,

$$
\left.\left.\mu(n, q)=\left[q^{(n+1) / 2}+(-1)^{n}\right]\left[q^{n / 2}-(-1)^{n}\right)\right] /(q-1)\right],
$$

it is possible to formulate the following proposition.

2000 Mathematics Subject Classification. Primary: 11E39, 51E20, Secondary: 05B25, 05B30.

Key words and phrases. Hermitian surfaces, intersection numbers, finite projective spaces, point-line configurations.

The present research was performed within the activity of G.N.S.A.G.A. of the Italian INDAM with the financial support of the Italian Ministry M.I.U.R., project "Strutture geometriche, combinatorica e loro applicazioni", 2003-04. 
Proposition 2. Let $\mathcal{H}_{1}, \mathcal{H}_{2}$ be two non-degenerate Hermitian varieties in $\mathrm{PG}(\mathrm{n}, \mathrm{q})$, and let $\left(r_{1}, \ldots, r_{n}\right)$ be the rank sequence of the pencil $\Gamma\left(\mathcal{H}_{1}, \mathcal{H}_{2}\right)$. Then,

$$
\begin{aligned}
\left|\mathcal{H}_{1} \cap \mathcal{H}_{2}\right|= & \eta_{n}(\Gamma, q)= \\
\frac{1}{\sqrt{q}(q-1)}\left\{\left(1-q^{n+1}\right)+\sum_{i=1}^{n} r_{i}\left[(q \sqrt{q} \mu(i-1, q)+1)\left(q^{n+1-i}-1\right)\right.\right. & -(q-1) \mu(n, q)]\}+\left(1+\frac{1}{\sqrt{q}}\right) \mu(n, q) .
\end{aligned}
$$

Table 1 outlines the possible intersection sizes for any two non-degenerate Hermitian surfaces in $\mathrm{PG}(3, \mathrm{q})$. All cases are possible.

\begin{tabular}{cccl}
\hline \hline$r_{1}$ & $r_{2}$ & $r_{3}$ & $\eta_{3}(\Gamma, q)$ \\
\hline 0 & 0 & 0 & $(q+1)^{2}$ \\
0 & 0 & 1 & $(q+\sqrt{q}+1)(q-\sqrt{q}+1)$ \\
0 & 0 & 2 & $\left(q^{2}+1\right)$ \\
0 & 0 & 3 & $q^{2}-q+1$ \\
0 & 0 & 4 & $(q-1)^{2}$ \\
0 & 1 & 0 & $q^{2}+q \sqrt{q}+q+1$ \\
0 & 1 & 1 & $q^{2}+q \sqrt{q}+1$ \\
0 & 1 & 2 & $(\sqrt{q}+1)(q \sqrt{q}-q+1)$ \\
0 & 2 & 0 & $(\sqrt{q}+1)(q \sqrt{q}+q-\sqrt{q}+1)$ \\
1 & 0 & 0 & $q \sqrt{q}+q+1$ \\
1 & 0 & 1 & $q \sqrt{q}+1$ \\
\hline
\end{tabular}

TABLE 1. Possible intersection numbers for Hermitian surfaces: non-degenerate pencil.

In the rest of this paper we shall usually write just $\Gamma$ for $\Gamma\left(\mathcal{H}_{1}, \mathcal{H}_{2}\right)$ when no ambiguity might arise and we shall denote by $\mathcal{E}$ the intersection $\mathcal{H}_{1} \cap \mathcal{H}_{2}$.

\section{DESCRIPTION OF THE CONFIGURATIONS}

3.1. Pencils with a degenerate surface of rank 1. The simplest case to consider is when the linear system $\Gamma$ contains a degenerate surface $\mathcal{C}$ of rank 1 , that is a plane repeated $q+1$ times.

In this case, the intersection is either a degenerate or non-degenerate Hermitian, according as $\mathcal{C}$ is secant or tangent to all the other surfaces in the pencil. It follows directly from Table 1 that the former case occurs when $\Gamma$ contains also a surface of rank 3, a Hermitian cone, whereas the latter is possible only if all the surfaces in $\Gamma \backslash\{\mathcal{C}\}$ are non-degenerate.

3.2. Pencils whose degenerate surfaces have all rank 2. A Hermitian surface $\mathcal{P}$ of rank 2 is a set of $\sqrt{q}+1$ planes through a line, the radical of $\mathcal{P}$. In the following propositions we distinguish several cases.

Proposition 3. Suppose that $\Gamma$ contains exactly one degenerate surface $\mathcal{P}$ of rank $2 . \quad$ Then, either 1,2 or $(\sqrt{q}+1)$ components of $\mathcal{P}$ are degenerate Hermitian curves.

Proof. The radical of $\mathcal{P}$ meets $\mathcal{E}$ in either $1, \sqrt{q}+1$ or $q+1$ points. Let $n=|\operatorname{rad} \mathcal{P} \cap \mathcal{E}|$ and denote by $v_{2}$ the number of components of $\mathcal{P}$ which meet $\mathcal{E}$ in a degenerate Hermitian curve. 
(1) $n=1$. Then,

$$
q^{2}+q \sqrt{q}+q+1=v_{2}(q \sqrt{q}+q)+\left(\sqrt{q}+1-v_{2}\right) q \sqrt{q}+1
$$

hence, $v_{2}=1$.

(2) $n=\sqrt{q}+1$. Then,

$$
q^{2}+q \sqrt{q}+q+1=v_{2}(q \sqrt{q}+q-\sqrt{q})+\sqrt{q}\left(\sqrt{q}+1-v_{2}\right)(q-1)+\sqrt{q}+1 ;
$$

hence, $v_{2}=2$.

(3) $n=q+1$. Then,

$$
q^{2}+q \sqrt{q}+q+1=v_{2}(q \sqrt{q})+q\left(\sqrt{q}+1-v_{2}\right)(\sqrt{q}-1)+q+1 ;
$$

hence, $v_{2}=\sqrt{q}+1$.

Observe that in general, if $\mathcal{P}$ and $\mathcal{P}^{\prime}$ are any two degenerate surfaces in $\Gamma$, then $\mathcal{R}=\operatorname{rad} \mathcal{P} \cap$ $\operatorname{rad} \mathcal{P}^{\prime}=\emptyset$ since, otherwise, any point $V \in \mathcal{R}$ would be singular for all the surfaces of $\Gamma$. Suppose now that there are two distinct Hermitian surfaces $\mathcal{P}$ and $\mathcal{P}^{\prime}$ both of rank 2 in $\Gamma$; the previous observation proves that $\operatorname{rad} \mathcal{P}$ and $\operatorname{rad} \mathcal{P}^{\prime}$ have to be mutually skew. Furthermore, both $\operatorname{rad} \mathcal{P}$ and $\operatorname{rad} \mathcal{P}^{\prime}$ meet any non-degenerate surface in $\Gamma$ in $(\sqrt{q}+1)$ points. Thus, we obtain the following proposition.

Proposition 4. The intersection of two non degenerate Hermitian surfaces $\mathcal{H}_{1}, \mathcal{H}_{2}$ spawning a pencil with $r_{2}=2$ is the union of all generators of $\mathcal{H}_{1}$ which pass through two skew $(\sqrt{q}+1)-$ secants.

3.3. Pencils whose degenerate surfaces have rank 2 and 3. Given a Hermitian cone $\mathcal{C}$ of vertex $V$ and a non-degenerate Hermitian variety $\mathcal{H}$, we denote by $\Gamma^{\prime}(\mathcal{C}, \mathcal{H})$ the $\mathrm{GF}(\sqrt{\mathrm{q}})$-linear system of Hermitian curves generated by $\mathcal{C}^{\prime}=\mathcal{C} \cap \pi$ and $\mathcal{H}^{\prime}=\mathcal{H} \cap \pi$, where $\pi$ is the polar plane of $V$ with respect to $\mathcal{H}$.

Lemma 5. Let $\mathcal{C}_{1}$ and $\mathcal{C}_{2}$ be two distinct Hermitian cones of vertices respectively $V_{1}$ and $V_{2}$. Assume that the pencil $\Gamma\left(\mathcal{C}_{1}, \mathcal{C}_{2}\right)$ contains at least a non-singular surface and that $V_{1} \notin \mathcal{E}$. Then, $V_{2}$ belongs to the polar plane of $V_{1}$ with respect to any non-degenerate Hermitian surface in $\Gamma$.

Proof. Fix a non-degenerate Hermitian surface $\mathcal{H} \in \Gamma$ and let $\pi$ be the polar plane of $V_{1}$ with respect to $\mathcal{H}$. Since $V_{1} \notin \mathcal{H}$, the plane $\pi$ cuts a non-singular Hermitian curve on $\mathcal{H}$. Suppose $V_{2} \notin \pi$; then the line $V_{1} V_{2}$ would meet $\mathcal{H}$ in $\sqrt{q}+1$ points. On the other hand, $V_{1} V_{2}$ meets $\mathcal{C}_{1}$ and $\mathcal{C}_{2}$ in either 1 or $q+1$ points - a contradiction. It follows that $V_{2} \in \pi$ and $\left|V_{1} V_{2} \cap \mathcal{E}\right| \leq 1$.

Lemma 6. Take $\mathcal{C}$ to be a Hermitian cone of vertex $V$ and $\mathcal{H}$ to be a non-degenerate Hermitian surface; let also $\pi$ be the polar plane of $V$ with respect to $\mathcal{H}$ and let $\Gamma=\Gamma(\mathcal{C}, \mathcal{H})$. Then,

(1) if $V \notin \mathcal{H}$,

(2) if $V \in \mathcal{H}$,

$$
\eta_{3}(\Gamma, q)=q^{2}+q \sqrt{q}+\sqrt{q}+1-\eta_{2}\left(\Gamma^{\prime}, q\right) \sqrt{q}
$$

$$
\eta_{3}(\Gamma, q)=q^{2}-q+|\pi \cap \mathcal{E}| .
$$

Proof. Let $\mathcal{H}^{\prime}=\mathcal{H} \cap \pi$ and $\mathcal{C}^{\prime}=\mathcal{C} \cap \pi$. Take $h=\eta_{2}\left(\Gamma^{\prime}, q\right)$. Observe that any line through $V$ tangent to $\mathcal{H}$ is of the form $P V$ with $P \in \mathcal{H}^{\prime}$. If $V \notin \mathcal{H}$, every line through $V$ meets $\mathcal{H}$ in either 1 or $\sqrt{q}+1$ points; on the other hand, exactly $h$ generators of $\mathcal{C}$ are tangent to $\mathcal{H}$, whence it follows

$$
\eta_{3}(\Gamma, q)=h+(q \sqrt{q}+1-h)(\sqrt{q}+1) .
$$

Assume $V \in \mathcal{H}$. Hence, $\pi$ is the tangent plane to $\mathcal{H}$ at $V$ and $\mathcal{H}^{\prime}$ consists of $\sqrt{q}+1$ lines through $V$. However, $\mathcal{C}^{\prime}$ consists of either 1 line or a degenerate Hermitian curve. In the former case we 
would have $\eta_{3}(\Gamma, q)=q^{2}+q+1$, which is not possible. Hence, $\mathcal{C}^{\prime}$ is the union of $\sqrt{q}+1$ lines through $V$ and

$$
\eta_{3}(\Gamma, q)=\sqrt{q}(q \sqrt{q}-\sqrt{q})+|\pi \cap \mathcal{E}| .
$$

Observe that in this case, all the curves in the linear system $\Gamma$ are degenerate.

Using the cardinality formula of Proposition 2] together with Lemma [6] it is possible to reconstruct the rank sequence of $\Gamma(\mathcal{C}, \mathcal{H})$ from the rank sequence of $\Gamma^{\prime}$.

Lemma 7. Assume $\Gamma$ to contain at least one cone $\mathcal{C}$ of vertex $V \notin \mathcal{E}$, and let the rank sequence of $\Gamma^{\prime}$ be $\left(r_{1}^{\prime}, r_{2}^{\prime}\right)$. Then, the rank sequence of $\Gamma$ is $\left(0, r_{1}^{\prime}, r_{2}^{\prime}+1\right)$.

In fact, it is possible to describe in an accurate way the actual configuration $\mathcal{E} \cap \pi$ in terms of the seven classes of [2]. We shall denote such classes as in [3].

Proposition 8. Let $\Gamma$ be a $\mathrm{GF}(\sqrt{\mathrm{q}})$-pencil of Hermitian surfaces with rank sequence $\left(0, r_{1}^{\prime}, r_{2}^{\prime}+1\right)$. Then, the base configuration $\mathcal{E}^{\prime}=\mathcal{E} \cap \pi$ is uniquely determined.

Proof. The cardinality of $\mathcal{E}^{\prime}$ is determined by Lemma 7 Observe that 5 of the 7 classes of [2 are uniquely determined by their rank sequence. However, both class III and IV correspond to the same rank sequence $\left(r_{1}^{\prime}, r_{2}^{\prime}\right)=(0,1)$. By Lemma $\mathbf{Z} \Gamma$ has necessarily rank sequence $(0,0,2)$ and $|\mathcal{E}|=q^{2}+1$. Denote then by $\mathcal{C}_{1}$ and $\mathcal{C}_{2}$ the two distinct Hermitian cones of $\Gamma$ and assume they have respectively vertices $V_{1}$ and $V_{2}$. There are two possibilities for $\mathcal{E}^{\prime}=\mathcal{E} \cap \pi$ :

(1) $\mathcal{E}^{\prime}$ belongs to class III, that is $\mathcal{E}^{\prime}$ consists of $\sqrt{q}-1$ sublines, all disjoint, and 2 more points;

(2) $\mathcal{E}^{\prime}$ belongs to class IV, that is $\mathcal{E}^{\prime}$ consists of $\sqrt{q}$ sublines, which meet all in a point $P$.

The former case occurs if $V_{2} \notin \mathcal{E}$. If $\mathcal{E}^{\prime}$ belongs to class IV, then $V_{2}=P \in \mathcal{E}$.

Proposition 9. Let $\Gamma$ be a non-degenerate linear system of Hermitian surfaces with $r_{2}(\Gamma)=$ $r_{3}(\Gamma)=1$. Then, $\mathcal{E}$ is either the union of $\sqrt{q}+1$ non-degenerate Hermitian curves all with a point in common, or the union of $\sqrt{q}$ non-degenerate Hermitian curves and a degenerate Hermitian curve, all sharing $a(\sqrt{q}+1)$-secant.

Proof. Let $\mathcal{P}$ and $\mathcal{C}$ be respectively the only surface of rank 2 and the only Hermitian cone in $\Gamma$. Let also $L=\operatorname{rad} \mathcal{P}$ and $V$ be the vertex of $\mathcal{C}$. Observe that $l=|L \cap \mathcal{E}| \in\{1, \sqrt{q}+1\}$.

(1) $l=1$. Let $M$ be the point of intersection of $\mathcal{C}$ and $L$; clearly $M \neq V$. If $V \in \mathcal{E}$, then there is a component $\pi$ of $\mathcal{P}$ such that $V \in \pi$. However, in this case $\mathcal{C} \cap \pi=P M$. However, this cannot be a plane section of a non-degenerate Hermitian surface; hence, it follows that $V \notin \mathcal{E}$. This being the case, all the $\sqrt{q}+1$ sections cut on $\mathcal{C}$ by $\mathcal{P}$ are non-degenerate Hermitian curves having the point $M$ in common.

(2) $l=\sqrt{q}+1$ : Let $v_{1}$ be the number of the components of $\mathcal{P}$ which meet $\mathcal{C}$ in a degenerate Hermitian curve. Observe that $v_{1} \leq 1$ and equality occurs if and only if $V \in \mathcal{E}$. Since,

$$
\left(q^{2}+q \sqrt{q}+1\right)=(\sqrt{q}+1)+\left(\sqrt{q}+1-v_{1}\right)(q \sqrt{q}-\sqrt{q})+v_{1}\left(q \sqrt{q}+q^{2}-\sqrt{q}\right),
$$

we get $v_{1}=1$ and $V \in \mathcal{E}$.

Proposition 10. Let $\Gamma$ be a non-degenerate linear system of Hermitian surfaces with $r_{2}(\Gamma)=1$ and $r_{3}(\Gamma)=2$. Then, $\mathcal{E}$ is the union of $\sqrt{q}+1$ non degenerate Hermitian curves, all sharing a chord.

Proof. Let $\mathcal{P}$ be the only Hermitian surface or rank 2 in $\Gamma$ and denote by $\mathcal{C}_{1}, \mathcal{C}_{2}$ be the two Hermitian cones. As before, denote by $V_{1}$ and $V_{2}$ the vertices of respectively $\mathcal{C}_{1}$ and $\mathcal{C}_{2}$ and define $L=\operatorname{rad} \mathcal{P}$ and $l=|L \cap \mathcal{E}|$. Either $l=1$ or $l=\sqrt{q}+1$. If it were $l=1$, then we would obtain that $\mathcal{E}$ is the union of $\sqrt{q}+1$ non-degenerate Hermitian curves, all with a point 
in common. However, this is a contradiction because of Proposition 2. Assume then $l=\sqrt{q}+1$ and denote by $v_{1}$ the number of components of $\mathcal{P}$ meeting $\mathcal{E}$ in a degenerate Hermitian curve. Then,

$$
(\sqrt{q}+1)(q \sqrt{q}-q+1)=(\sqrt{q}+1)+\left(\sqrt{q}+1-v_{1}\right)(q \sqrt{q}-\sqrt{q})+v_{1}\left(q \sqrt{q}+q^{2}-\sqrt{q}\right) .
$$

This is possible only if $v_{1}=0$, that is, $\mathcal{E}$ is the union of $\sqrt{q}+1$ non-degenerate Hermitian curves, all sharing a chord.

3.4. Pencils whose degenerate surfaces have all rank 3. All the pencils considered in this section contains at least a cone $\mathcal{C}$. We shall denote by $s_{1}, s_{2}$ and $s_{3}$ the number of generators of $\mathcal{C}$ meeting $\mathcal{E}$ in respectively $q+1, \sqrt{q}+1$ or 1 points.

Lemma 11. Let $\Gamma$ contain at least 3 distinct cones $\mathcal{C}_{1} \ldots \mathcal{C}_{3}$ of vertices $V_{1} \ldots V_{3}$. Then, either the vertices of all the cones in $\Gamma$ are collinear or at most one of them is in $\mathcal{E}$.

Proof. Suppose $V_{1}, V_{2} \in \mathcal{E}$; then, $V_{1} V_{2} \subseteq \mathcal{E}$. However, for $V_{1} V_{2}$ to be a subset of $\mathcal{E}$, it is necessary for it to be a generator of $\mathcal{C}_{3}$ also. It follows $V_{3} \in V_{1} V_{2}$.

Observe that when $\Gamma$ contains at least two Hermitian cones, the number of lines in $\mathcal{E}$ is at most 1 .

Proposition 12. If $\Gamma$ contains 4 Hermitian cones, then none of the vertices of such cones belongs to $\mathcal{E}$ and exactly $\sqrt{q}(q-\sqrt{q}-2)$ generators of any cone meet $\mathcal{E}$ in $(\sqrt{q}+1)$ points, the remaining $(\sqrt{q}+1)^{2}$ being tangent lines.

Proof. By Proposition 2, $|\mathcal{E}|=(q-1)^{2}$. Suppose $V_{1}, V_{2}, V_{3}, V_{4} \in \mathcal{E}$. Then,

$$
(q-1)^{2}=(q+1)+s_{2} \sqrt{q}+\left(q \sqrt{q}-s_{2}\right),
$$

that is $q\left(q^{2}-\sqrt{q}-3\right)=s_{2}(\sqrt{q}-1)$, a contradiction since $(\sqrt{q}-1)$ does not divide $q^{2}-\sqrt{q}-3$. If $V_{1} \in \mathcal{E}$ while $V_{2}, V_{3}, V_{4} \notin \mathcal{E}$. Observe that a generator of $\mathcal{C}_{1}$ either meets a non-degenerate surface $\mathcal{H}$ is 1 or in $\sqrt{q}+1$ points. Then,

$$
(q-1)^{2}=s_{2} \sqrt{q}+1,
$$

which gives $s_{2}=q \sqrt{q}-2 \sqrt{q}$, that is $2 \sqrt{q}+1$ generators of $\mathcal{C}$ meet $\mathcal{H}$ in $V$ only and all these generators lie in the tangent plane to $\mathcal{H}$ at $V$. However, the number of generators of $\mathcal{C}$ on any plane is at most $\sqrt{q}+1$, which gives a contradiction. It follows that $\mathcal{E}$ does not contain the vertex of any cone in $\Gamma$ and

$$
(q-1)^{2}=s_{2}(\sqrt{q}+1)+\left(q \sqrt{q}+1-s_{2}\right),
$$

that is, $s_{2}=\sqrt{q}(q-\sqrt{q}-2)$, which gives the result.

Proposition 13. Suppose $\Gamma$ to contain exactly 3 cones $\mathcal{C}_{1}, \mathcal{C}_{2}, \mathcal{C}_{3}$. Then, there are two possibilities:

(1) $V_{1}, V_{2}, V_{3} \notin \mathcal{E}$ : then $\sqrt{q}(q-\sqrt{q}-1)$ components of each cone are $(\sqrt{q}+1)$-secants to any non-degenerate Hermitian surface in $\Gamma$;

(2) $V_{1} \in \mathcal{E}$ but $V_{2}, V_{3} \notin \mathcal{E}:$ then $\sqrt{q}(q-1)$ generators of the cone $\mathcal{C}_{1}$ meet $\mathcal{E}$ in $(\sqrt{q}+1)$ points, the remaining intersecting $\mathcal{E}$ in $V_{1}$ only; the number of generators of the cones $\mathcal{C}_{2}$ and $\mathcal{C}_{3}$ meeting $\mathcal{E}$ in $(\sqrt{q}+1)$ points is $q \sqrt{q}-q-\sqrt{q}$, the others meeting $\mathcal{E}$ in distinct points;

Proof. The cardinality of $\mathcal{E}$ is $q^{2}-q+1$.

(1) Since $V_{1} \notin \mathcal{E}$,

$$
q^{2}-q+1=s_{2}(\sqrt{q}+1)+\left(q \sqrt{q}-s_{2}+1\right) .
$$

Hence, there are $\sqrt{q}(q-\sqrt{q}-1)$ components of $\mathcal{C}_{1}$ are meeting $\mathcal{E}$ in $(\sqrt{q}+1)$ points, the remaining $q+\sqrt{q}+1$ being tangent to any surface. 
(2) Since $V_{1} \in \mathcal{E}$, each generator of $\mathcal{C}_{1}$ meets $\mathcal{E}$ in either 1 or $\sqrt{q}+1$ points. We get

$$
q^{2}-q+1=1+t \sqrt{q} \text {. }
$$

It follows that $\sqrt{q}(q-1)$ generators through $V$ meet $\mathcal{E}$ in $\sqrt{q}+1$ points. Consider now another cone $\mathcal{C}_{2} \in \Gamma$. Since $V_{2} \notin \mathcal{E}$, we get

$$
q^{2}-q+1=s_{2}(\sqrt{q}+1)+\left(q \sqrt{q}+1-s_{2}\right) ;
$$

hence $s_{2}=q \sqrt{q}-q-\sqrt{q}$.

Suppose now $V_{1}, V_{2} \in \mathcal{E}$. Then, the line $V_{1} V_{2}$ is a generator of any surface $\mathcal{H} \in \Gamma$ and we have

$$
q^{2}-q+1=q+1+s_{2} \sqrt{q} .
$$

It follows that $s_{2}=q \sqrt{q}-2 \sqrt{q}$. This gives that there should be $2 \sqrt{q}+1>\sqrt{q}+1$ generators through $V_{1}$ meeting $\mathcal{H}$ in $V_{1}$ only - a contradiction.

Proposition 14. Assume that the pencil $\Gamma$ contains exactly two cones $\mathcal{C}_{1}, \mathcal{C}_{2}$ of respectively vertices $V_{1}$ and $V_{2}$. Then, one of the following possibilities holds:

(1) both $V_{1}, V_{2} \in \mathcal{E} ;$ then, $\mathcal{E}$ contains the line $V_{1} V_{2} ; q(\sqrt{q}-1)$ components of each cone meet $\mathcal{E}$ in $\sqrt{q}+1$ points.

(2) $V_{1} \in \mathcal{E}$, while $V_{2} \notin \mathcal{E} ; \mathcal{E}$ does not contain any line; $q \sqrt{q}$ components of $\mathcal{C}_{1}$ and $q(\sqrt{q}-1)$ components of $\mathcal{C}_{2}$ meet $\mathcal{E}$ in $\sqrt{q}+1$ points.

(3) $V_{1}, V_{2} \notin \mathcal{E}$ belong to $\mathcal{E} ; q(\sqrt{q}-1)$ components of each cone meet $\mathcal{E}$ in $(q+1)$ points.

Proof. Let $V$ be the vertex of any cone $\mathcal{C}$ in $\Gamma$. Observe that for $V \notin \mathcal{E}$,

$$
q^{2}+1=s_{2}(\sqrt{q}+1)+\left(q \sqrt{q}+1-s_{2}\right) ;
$$

hence, $s_{2}=q \sqrt{q}-q$. On the other hand, if $V \in \mathcal{E}$

$$
q^{2}+1=s_{1} q+s_{2} \sqrt{q}+1 ;
$$

hence, $s_{2}=\sqrt{q}\left(q-s_{1}\right)$. The result now follows from $s_{1} \leq 1$.

Proposition 15. Assume that the only degenerate surface in the pencil $\Gamma$ is a cone $\mathcal{C}$. Then, either

(1) $V \notin \mathcal{E}$ and $\sqrt{q}(q-\sqrt{q}+1)$ components of $\mathcal{C}$ are meet $\mathcal{E}$ in $\sqrt{q}+1$ points, or

(2) $V \in \mathcal{E}$ and $\mathcal{E}$ contains at least a line.

Proof.

(1) If $V \notin \mathcal{E}$, then $s_{1}=0$ and

$$
q^{2}+q+1=s_{2}(\sqrt{q}+1)+\left(q \sqrt{q}+1-s_{2}\right) ;
$$

hence, $s_{2}=\sqrt{q}(q-\sqrt{q}+1)$.

(2) If $V \in \mathcal{E}$,

$$
q^{2}+q+1=s_{1} q+s_{2} \sqrt{q}+1
$$

hence, $s_{2}=\sqrt{q}\left(q+1-s_{1}\right)$. Since the total number of components of $\mathcal{C}$ is $q \sqrt{q}+1$, it follows that $s_{2} \leq q \sqrt{q}+1$ and $s_{1} \geq 1$.

Suppose that $\Gamma$ contains exactly one Hermitian cone whose vertex belongs to $\mathcal{E}$, and let $\pi$ be the tangent plane at $P$ to a non-degenerate Hermitian surface in $\Gamma$. Clearly, all generators in $\mathcal{E}$ lie in $\pi$; furthermore $s_{1} \in\{1,2, \sqrt{q}+1\}$. Consequently, $s_{2} \in\{q \sqrt{q}, q \sqrt{q}-\sqrt{q}, q \sqrt{q}-q\}$. We observe also that if $s_{1}>1$, then all non-degenerate Hermitian surfaces in $\Gamma$ share the same tangent plane at $P$. 


\section{REFERENCES}

[1] J.W.P. Hirschfeld, Projective geometries over finite fields, Oxford University Press, 1998

[2] B.C. Kestenband, Unital intersections in finite projective planes, Geom. Dedicata 11 (1981), no. 1, $107-117$.

[3] L. Giuzzi, Collineation groups of the intersection of two classical unitals, J. Combin Designs 9 (2001); 445459.

[4] L. Giuzzi, Hermitian varieties over finite fields, DPHIL Thesis, University of Sussex.

Dipartimento di Matematica, Facoltà di Ingegneria, Università degli studi di Brescia, via Valotti 9, 25133 Brescia, Italy. 